Psychother Psychosom 2012;81:383-385

DOI: $10.1159 / 000336873$

\section{Effectiveness of Cognitive-Behavioral Therapy Addition to Pharmacotherapy in Resistant Obsessive-Compulsive Disorder: A Multicenter Study}

Umberto Albert ${ }^{\mathrm{a}}$, Andrea Aguglia ${ }^{\mathrm{a}}$, Filippo Bogetto ${ }^{\mathrm{a}}$, Luca Cieri ${ }^{\mathrm{b}}$, Marinella Daniele ${ }^{\mathrm{b}}$, Giuseppe Maina ${ }^{\mathrm{a}}$, Roberta Neccib, Anita Parena $^{\mathrm{b}}$, Loretta Salvati ${ }^{\mathrm{b}}$, Antonio Tundo ${ }^{\mathrm{b}}$

a Department of Neuroscience, Anxiety and Mood Disorders Unit, University of Turin, Turin, and ${ }^{\mathrm{b}}$ Istituto di Psicopatologia, Rome, Italy

Available treatments for adult patients with obsessive-compulsive disorder (OCD) [1] are cognitive-behavioral therapy (CBT) and serotonin reuptake inhibitors (SRIs). Partial response to these treatments, however, is very frequent; the extant literature indicates that, when the response to SRIs is partial, augmentation with antipsychotics [2-7] may provide additional benefit. Antipsychotics' side effects, mainly the metabolic ones [8], however, limit this option. The second strategy consists of sequentially adding CBT; the efficacy of this strategy is suggested by open-label reports [9-14] and a single randomized, controlled trial [15].

Randomized, controlled studies have strong internal validity, but the generalizability of their findings to the real word could be limited by several factors $[16,17]$. In the study by Simpson et al. [15], for example, comorbid diagnoses were allowed only if secondary; mania, psychosis, suicidal ideation, and substance abuse/ dependence in the previous 6 months were exclusion criteria. Moreover, ERP protocol consisted of 17 twice-weekly sessions (90-120 min each), daily homework assignments, and betweensession phone calls and included $\geq 2$ sessions in the patients' home. One might ask if patients in the real world can follow this intensive CBT format. So, it could be useful for clinicians to know if the results of Simpson et al. [15] have been confirmed in large multicenter effectiveness studies.

The aim of this prospective, multicenter study was to investigate the effectiveness of routinely delivered CBT as an adjunct to medication in real-world OCD patients with incomplete response to medication.

Patients were recruited from the Department of Neuroscience, University of Turin, and the Istituto di Psicopatologia in Rome, Italy. Eligible participants were outpatients, aged $\geq 18$ years, with a primary DSM-IV (SCID-I) diagnosis of OCD, a Y-BOCS total score $\geq 16$, and incomplete response to $\geq 1$ adequate, prospective SRI trial. We included patients with partial but incomplete response (Y-BOCS reduction $<25 \%$ ) to at least 12 weeks of: $\geq 40 \mathrm{mg} /$ day of citalopram, fluoxetine, and paroxetine, $\geq 100 \mathrm{mg}$ /day of clomipramine, $\geq 20 \mathrm{mg} /$ day of escitalopram, $\geq 200 \mathrm{mg} /$ day of fluvoxamine and sertraline, and $\geq 250 \mathrm{mg} /$ day of venlafaxine. Exclusion criteria were a lifetime diagnosis of schizophrenia or other psychotic disorders, mental retardation, or an organic brain syndrome. All patients gave written informed consent, and a local ethical committee approved the research project.

Patients maintained the same drug and dosage while CBT was added (exposure and response prevention; cognitive therapy and other ad hoc interventions were used when necessary). Patients were treated in a naturalistic setting in the sense that manualized guidelines [18] were adapted to each patient by taking due account of the insight level into the senselessness of OCD symptoms, treatment adherence, and the presence of Axis I disorders. The therapist and the patient scheduled therapy sessions flexibly and jointly. CBT duration was not fixed in advance.

The primary outcome was Y-BOCS change from the beginning of CBT to the endpoint. CGI-S and GAF scores (although poorly validated) were also used. Response was defined as a Y-BOCS decrease $\geq 25 \%$ and remission as a Y-BOCS decrease $\geq 25 \%$ and $Y$-BOCS $<16$ posttreatment [19]. Predictors of the percentage of Y-BOCS reduction at T2 were examined with independent $t$ tests for dichotomous variables and Pearson's correlations for continuous variables. Significant variables were then entered into a stepwise multiple linear regression analysis.

One hundred nineteen patients were enrolled; $68.1 \%$ of them had $\geq 1$ lifetime comorbid disorder, and $28.6 \%$ bipolar disorder. All patients were currently taking $\geq 1$ SRI; $19.3 \%$ were taking two SRIs, and $34.5 \%$ were taking SRI plus atypical antipsychotic. The mean duration of the current treatment was 7.13 months, and most patients (82.4\%) received an adequate SRI dose for more than the required minimum time of 12 weeks. The number of lifetime ineffective trials, including the current one, was 2.78. Nineteen patients dropped out before T1, and 22 dropped out between T1 and T2; the cumulative proportion of dropouts was $34.5 \%$. Table 1 shows the variation of mean Y-BOCS, CGI-S, and GAF scores across time. The mean reduction in Y-BOCS score was $17.9 \%$ at $\mathrm{T} 1$ and $29.3 \%$ at $\mathrm{T} 2$. Responder rates were $32.8 \%$ at $\mathrm{T} 1$ and $58 \%$ at $\mathrm{T} 2$; remitter rates were 15.1 and $31.1 \%$, respectively. A higher baseline GCI-S score, having had a previous hospitalization for OCD, and a longer duration on the current ineffective treatment independently predicted in the final regression analysis a lower reduction in the Y-BOCS total score.

Our multicenter, prospective, naturalistic study indicates that the positive results of the controlled study concerning the efficacy of CBT addition to medication in partial responder OCD patients [15] can be generalized, although to a lesser degree, to routine clinical practice. The demonstration of the effectiveness of CBT addition in this clinical population is of high clinical relevance.

\section{KARGER}

Fax +4161306 1234 E-Mail karger@karger.ch www.karger.com
2012 S. Karger AG, Basel

$0033-3190 / 12 / 0816-0383 \$ 38.00 / 0$

Accessible online at: www.karger.com/pps
Umberto Albert

Department of Neurosciences, Anxiety and Mood Disorders Unit

University of Turin, Via Cherasco 11

IT-10126 Torino (Italy)

Tel. +39011 633 5425, E-Mail umberto.albert@unito.it 


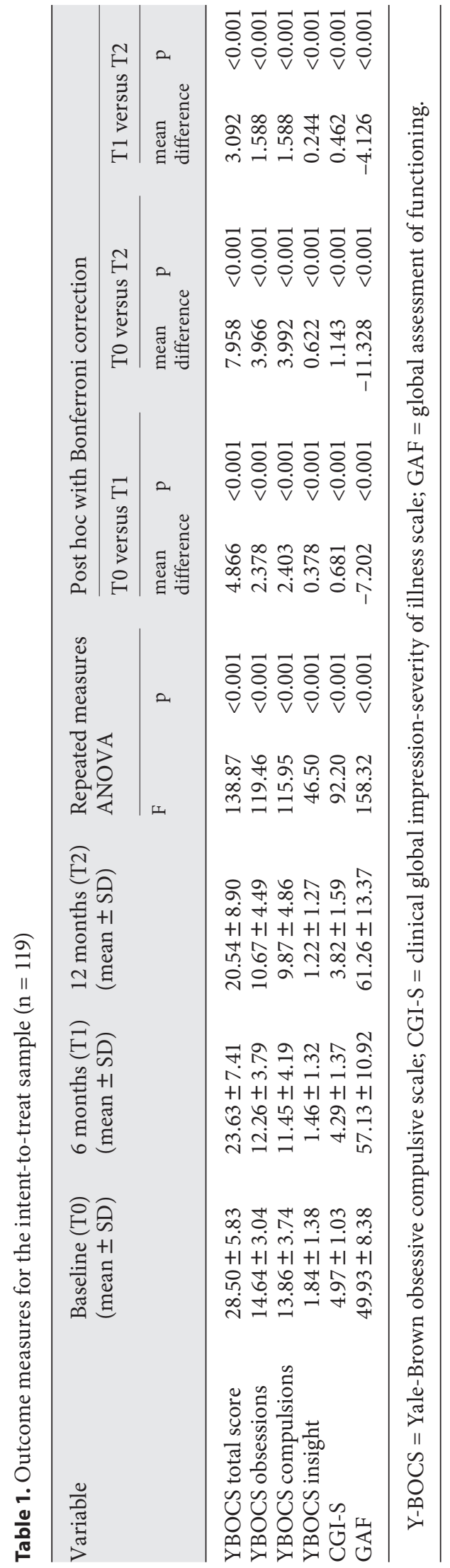

Our results are consistent with findings of previous open studies, with smaller samples $[9,10,12,13]$. Our response rates are lower than those of the controlled study (58\% at 12 months and $74 \%$ at 8 weeks, respectively) [15]. This difference could be explained by differences in the considered samples: we enrolled more severe patients than those participating in the controlled study in terms of the Y-BOCS total score (28.5 and 25.4, respectively), alcohol or substance use/dependence comorbidity (10.1 and $0 \%$, respectively), the number of previous ineffective treatments (2.78 and 1.5, respectively), and current use of combination/augmentation pharmacotherapy (53.8 and 8\%, respectively). Moreover, we cannot exclude that the lower response rate in our study may also be due to differences in treatment. CBT in our study was practiced in a naturalistic setting: manual procedures were adapted to each patient's needs, sessions were scheduled flexibly, treatment duration was not prefixed, and ERP exercise intensity was lower than in the controlled study. According to the typical use of CBT in the clinical setting, these changes in protocol were needed to enhance adherence to treatment and to test whether CBT addition was as effective in real-world patients as it was in the controlled trial.

Some caveats to our findings must be considered; there was no control group, participants were self-selected for inclusion, and there was no randomization across interventions. These design factors reflect the real-world nature of mental health services and represent, in our opinion, a major strength of our study as they give clinicians an idea of the exact amount of improvement to be expected in clinical practice.

\section{References}

1 American Psychiatric Association: Practice Guideline for the Treatment of Patients with Obsessive-Compulsive Disorder. Arlington, American Psychiatric Association, 2007.

$\checkmark 2$ Maina G, Albert U, Pessina E, Salvi V, Bogetto F: Antipsychotics in obsessive-compulsive disorder. Curr Psychiatry Rev 2005;1:292-301.

3 Bloch MH, Landeros-Weisenberger A, Kelmendi B, Coric V, Bracken MB, Leckman JF: A systematic review: antipsychotic augmentation with treatment refractory obsessive-compulsive disorder. Mol Psychiatry 2006;11:622-632.

4 Ipser JC, Carey P, Dhansey P, Fakier N, Seedat S, Stein DJ: Pharmacotherapy augmentation strategies in treatment-resistant anxiety disorders. Cochrane Database Syst Rev 2006;18:CD005473.

5 Skapinakis P, Papatheodorou T, Mavreas V: Antipsychotic augmentation of serotonergic antidepressants in treatment-resistant obsessivecompulsive disorder: a meta-analysis of the randomized controlled trials. Eur Neuropsychopharmacol 2007;17:79-93.

-6 Maina G, Albert U, Salvi V, Bogetto F: A review of current strategies for treatment resistant obsessive-compulsive disorder. Curr Drug Ther 2008;3:126-142.

7 Maina G, Pessina E, Albert U, Bogetto F: An 8-week, single-blind, randomized trial comparing risperidone versus olanzapine augmentation of serotonin reuptake inhibitors in treatment resistant obsessive-compulsive disorder. Eur Neuropsychopharmacol 2008;18:364-372.

8 Matsunaga H, Nagata T, Hayashida K, Ohya K, Kiriike N, Stein DJ: A long-term trial of the effectiveness and safety of atypical antipsychotic agents in augmenting SSRI-refractory obsessive-compulsive disorder. J Clin Psychiatry 2009;70:863-868.

-9 Simpson HB, Gorfinkle KS, Liebowitz MR: Cognitive-behavioral therapy as an adjunct to serotonin reuptake inhibitors in obsessive-compulsive disorder: an open trial. J Clin Psychiatry 1999;60:584-590.

$\checkmark 10$ Kampman M, Keijsers GP, Hoogduin CA, Verbraak MJ: Addition of cognitive-behavior therapy for obsessive-compulsive disorder patients non-responding to fluoxetine. Acta Psychiatr Scand 2002;106:314-319. 
11 Albert U, Maina G, Forner F, Bogetto F: Cognitive-behavioral therapy in obsessive-compulsive disorder patients partially unresponsive to SRIs. Eur Neuropsychopharmacol 2003;13(suppl 4):S357.

12 Tolin DF, Maltby N, Diefenbach DJ, Hannan SE, Worhunsky P: Cognitive-behavioral therapy for medication nonresponders with obsessivecompulsive disorder: a wait-list-controlled open trial. J Clin Psychiatry 2004;65:922-931.

13 Tundo A, Salvati L, Busto G, Di Spigno D, Falcini R: Addition of cognitive-behavioural therapy for nonresponders to medication for obsessive-compulsive disorder: a naturalistic study. J Clin Psychiatry 2007; 68:1552-1556.

14 Anand N, Sudhir PM, Math SB, Thennarasu K, Reddy YC: Cognitive behaviour therapy in medication non-responders with obsessive-compulsive disorder: a prospective 1-year follow-up study. J Anxiety Disord 2011;25:939-945.
15 Simpson HB, Foa EB, Liebowitz MR, Ledley DR, Huppert JD, Cahill S, Vermes D, Schmidt AB, Hembree E, Franklin M, Campeas R, Hahn CG, Petkova E: A randomized, controlled trial of cognitive-behavioral therapy for augmenting pharmacotherapy in obsessive-compulsive disorder. Am J Psychiatry 2008;165:621-630.

16 Kazdin A: Methodology, design, and evaluation in psychotherapy research; in Bergin AE, Garfield SL (eds): Handbook of Psychotherapy and Behaviour Change, ed 4. New York, Wiley, 1994, pp 19-71.

17 Margison FR, Barkham M, Evans C, McGrath G, Clark J, Audin K, Connell J: Measurement and psychotherapy. Br J Psychiatry 2000;177: 123-130.

18 Kozak MJ, Foa EB: Mastery of obsessive-compulsive disorder: a cognitive behavioral approach-therapist guide. San Antonio, The Psychological Corporation, 1997.

19 Pallanti S, Quercioli L: Treatment-refractory obsessive-compulsive disorder: methodological issues, operational definitions and therapeutic lines. Prog Neuropsychopharmacol Biol Psychiatry 2006;30:400-412. 